\title{
In-situ temperature monitoring with photoacoustics during photothermal therapy and perspectives for glioblastoma treatment monitoring
}

K. Metwally, C. Bastiancich, N. Dumas, F. Correard, A. Novell, et al.

K. Metwally, C. Bastiancich, N. Dumas, F. Correard, A. Novell, F. Chaspoul, N. Jones, G. Tselikov, A. Popov, A. Al-Kattan, A. V. Kabashin, B. Larrat, D. Braguer, S. Mensah, M.-A. Estève, A. Da Silva, "In-situ temperature monitoring with photoacoustics during photothermal therapy and perspectives for glioblastoma treatment monitoring," Proc. SPIE 11077, Opto-Acoustic Methods and Applications in Biophotonics IV, 1107709 (19 July 2019); doi: $10.1117 / 12.2527060$ 


\title{
In Situ Temperature Monitoring with PhotoAcoustics during Photothermal Therapy and Perspectives for Glioblastoma Treatment Monitoring
}

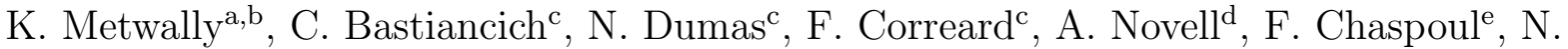 \\ Jones $^{\mathrm{b}}$, G. Tselikov ${ }^{\mathrm{f}}$, A. Popov ${ }^{\mathrm{f}}$, A. Al-Kattan ${ }^{\mathrm{f}}$, AV. Kabashin ${ }^{\mathrm{f}}$, B. Larrat ${ }^{\mathrm{d}}$, D. Braguer ${ }^{\mathrm{c}}$, S. \\ Mensah $^{\mathrm{b}}$, MA. Estève ${ }^{\mathrm{c}}$, and A. Da Silva ${ }^{\mathrm{a}}$ \\ aAix Marseille Univ, CNRS, Centrale Marseille, Institut Fresnel, Marseille, France \\ ${ }^{\mathrm{b}}$ Aix Marseille Univ, CNRS, Centrale Marseille, LMA, Marseille, France \\ cAix Marseille Univ, CNRS, UMR 7051 Institut Neurophysiopathologie, Marseille, France

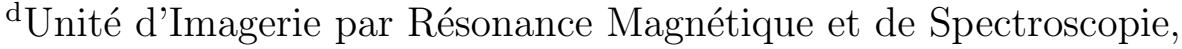 \\ CEA/DRF/I2BM/NeuroSpin, Gif-sur-Yvette, France \\ eAix Marseille Univ, CNRS, IRD, IMBE, Marseille, France \\ ${ }^{\mathrm{f}}$ Aix Marseille Univ, CNRS, LP3, Campus de Luminy, Marseille
}

\begin{abstract}
In situ temperature monitoring with photoacoustic measurements is introduced in an integrated setup, specifically designed for photothermotherapy treatmentof the glioblastoma, aided by nanoparticles and HIFU blood-brain barrier opening.
\end{abstract}

Keywords: PhotoAcoustics, Temperature, Photothermal Therapy

\section{INTRODUCTION}

Cancer statistics show an excessively high occurence ratio and mortality rate $(\sim 79 \%)$ due to brain cancer. Glioblastoma (GBM) is the most aggressive malignant brain tumor in adults. GBM is incurable because of its high invasiveness, chemoresistance and high tendency to form recurrences, even after surgical resection combined with radiotherapy and chemotherapy. ${ }^{1}$ Existing therapies including surgery, radiotherapy or chemotherapy fail to attenuate tumor growth. Even though the improvement to patient survival and mortality rates they provide, there is an urgent need for more efficient therapeutic strategies.

In this aim, a new multidisciplinary therapeutic strategy is proposed for treatment of GBM. The objective is to achieve photo-hyperthermia treatment and non-invasive local monitoring of temperature with photoacoustic (PA) thermometry. As an attempt to address the chemoradiation drawbacks and find a long-lasting therapy for GBM, metallic nanoparticles (NPs) have emerged as potential absorbing agents for photothermal therapy (PTT). ${ }^{2}$ Recently, laser-synthesized Titanium Nitride Nanoparticles (TiN NP) have emerged as new candidate, ${ }^{3}$ for which we have demonstrated in vitro (U87-MG GBM cell cultures) its low cytotoxicity, a good cell uptake and PTT effect. Furthermore, in order to promote the accumulation of the nanoparticles in the tumor, a beforehand reversible blood brain barrier (BBB) opening strategy using high intensity focused ultrasound (HIFU) induced microbubbles stable cavitation was implemented.

In the present study, the focus is made on the possibilities of in situ temperature monitoring during PTT. A fully integrated setup is presented. An in vivo study was conducted on 20 mice.

Further author information: (Send correspondence to A. Da Silva)

A. Da Silva: E-mail: anabela.dasilva@fresnel.fr, Telephone: +33491288482

Opto-Acoustic Methods and Applications in Biophotonics IV, edited by Vasilis Ntziachristos,

Roger Zemp, Proc. of SPIE-OSA Vol. 11077, 1107709 - (C) 2019 SPIE-OSA

CCC code: $1605-7422 / 19 / \$ 21 \cdot$ doi: $10.1117 / 12.2527060$

Proc. of SPIE-OSA Vol. 11077 1107709-1 


\section{TEMPERATURE MONITORING WITH PHOTOACOUSTICS}

The amplitude of the PA signal $p$ is proportional to the Grueneisen coefficient $\Gamma$, accounting for the thermodynamical, and consequently mechanical, properties of the medium: $p=\Gamma \mu_{a} \phi$, where $\mu_{a}$ is the optical absorption coefficient, $\phi$ is the local fluence. $\Gamma$ is a function of the thermal expansion coefficient, the speed of sound, and the specific heat capacity. Within a reasonable range of temperatures, it has been shown that $\Gamma$ varies linearly as a function of the temperature $T$ in most biological tissues: ${ }^{4} p(T)=\left[1-b\left(T-T_{o}\right)\right] p\left(T_{o}\right)$, where $T_{o}$ is the reference temperature and $b$ an experimental calibration factor.

\section{A FULLY INTEGRATED THERAPY AND TEMPERATURE MONITORING SETUP}

In our setup (Fig.1), three laser sources were aligned in a single optical fibre to achieve three main tasks: alignment, photothermal heating and photoacoustic signal generation. The last one uses a pulsed laser (Quantel, France, Nd:Yag, $1064 \mathrm{~nm}, 330 \mathrm{~mJ}, 5 \mathrm{~ns}, 10 \mathrm{~Hz}$, frequency doubled by a KTP crystal at $532 \mathrm{~nm}$ ) and focused transducers (diameter $25 \mathrm{~mm}$, focal depth $20 \mathrm{~mm}$, central frequency $1.5 \mathrm{MHz}$,Imasonic, France) in order to achieve real-time monitoring. The collected signal is driven into an oscilloscope (PICOSCOPE 5243A from PICO TECHNOLOGY, 2 Channel, $100 \mathrm{MHz}, 500 \mathrm{MSPS}, 64 \mathrm{Mpts}, 5.8 \mathrm{~ns}$ ) for signal analyses. A Matlab graphical user interface code was developed to control different instruments on the setup. The second laser source is a continuous wave (CW) laser diode (Thorlabs L808P1000MM, $808 \mathrm{~nm}, 1000 \mathrm{~mW}$ ) mounted on a TECooled Mount (Thorlabs, LDM90/M) in which the temperature and the current are controlled using Benchtop Laser Diode TEC Controller (Thorlabs, ITC4005, 5A/225W).
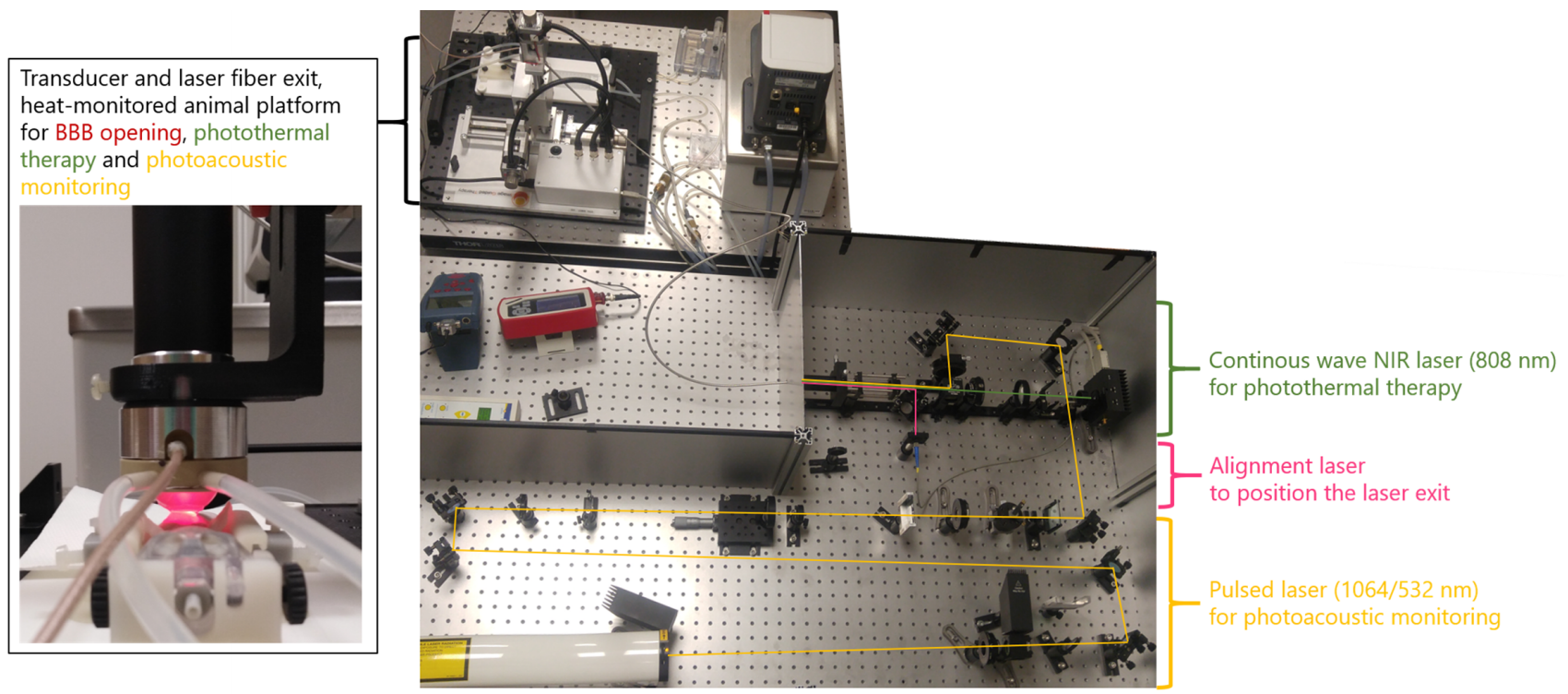

Figure 1. Integrated experimental setup.

\section{IN VIVO STUDY}

In vivo experiments were approved by the institutions Animal Care and Use Committee (CE14, Aix-Marseille Université) and performed following the French national regulation guidelines in accordance with EU Directive 2010/63/EU. Animals had free access to water and food. Six-week-old female NMRI nude mice (Envigo, France) were anesthetized by intraperitoneal injection of ketamine/xylazine (100 and $10 \mathrm{mg} / \mathrm{kg}$, respectively) and U87 MG cells were injected subcutaneously in their right flank $(2 \times 106$ cells/mouse). Tumors size was measured every other day using an electronic caliper and the volumes were calculated using the formula corresponding to a prolate ellipsoid: volume $=\pi / 6 \times$ length $\times$ widt $h^{2}$. 
When the tumors reached the volume of about $20 \mathrm{~mm}^{3}$, mice were randomized into 4 groups and treated as follows: Group 1: no treatment $(n=8)$; Group 2: laser irradiation $(n=7)$; Group 3: TiN NP intratumoral injection (30 L, $4 \mathrm{mg} / \mathrm{mL} ; \mathrm{n}=9)$; Group 4: TiN NP intratumoral injection (30 L, $4 \mathrm{mg} / \mathrm{mL}$ ) followed by laser irradiation $(\mathrm{n}=9)$. Animals of groups 2, 3 and 4 were anesthetized by intraperitoneal injection of ketamine/xylazine (100 and $10 \mathrm{mg} / \mathrm{kg}$, respectively) prior to treatment administration. For groups 2 and 4, animals were placed in dedicated temperature-controlled frame in prone position and the transducer was held using dedicated set-up and coupled to the tumor with a latex balloon filled with deionized and degassed water. The tumors were irradiated for 10 min by the CW NIR laser $(808 \mathrm{~nm})$ for PTT and by the pulsed laser $(532 \mathrm{~nm})$ for PA measurement. After the treatment, animals were monitored (body weight, physical signs of pain or distress) and tumors measured three times a week. Animals were sacrificed when the tumor reached $800 \mathrm{~mm}^{3}$.

The temperature evolution profile as a function of time of CW laser exposure clearly exhibits a linear increase of temperature within the tumor when the PTT is enhanced by the use of TiN NPs.

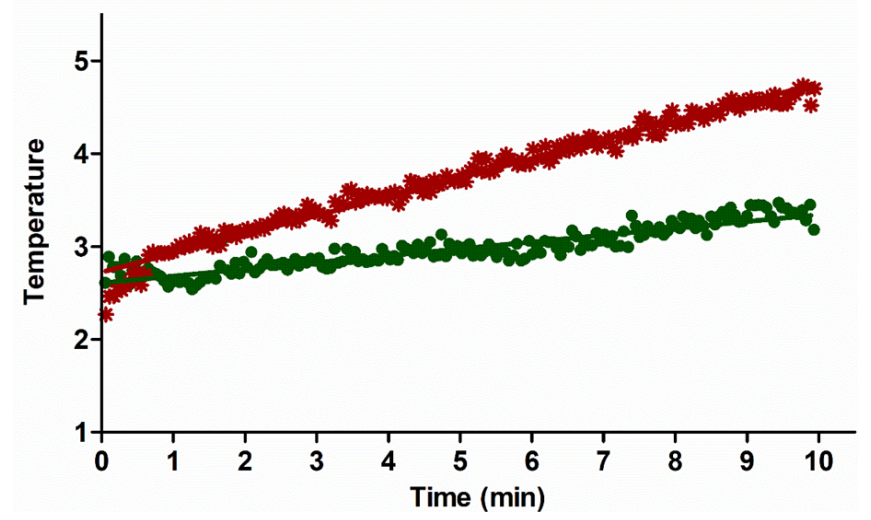

Figure 2. Evolution of the PA maximum intensity as a function of the PTT time treatment: green, without NPs

(Groupe 2), red, with (Groupe 4).

\section{CONCLUSION}

In the present study, an integrated PTT system, with temperature monitoring by PA, is presented. An animal study demonstrates the possibilities of in situ temperature monitoring during PTT. This control can be used as a real time monitoring of the optical radiation dose delivered locally at the site of the tumor.

\section{ACKNOWLEDGMENTS}

This project is developed in the field of the Glioblastoma Risk Attrition by Vectorized Thermotherapy - GRAVITY project (PC201613) funded by the ITMO Plan Cancer 20142019 INSERM program

\section{REFERENCES}

[1] Grossman, S. A. and Batara, J. F., "Current management of glioblastoma multiforme," Seminars in Oncology 31(5), 635 - 644 (2004). Brain Tumors.

[2] Riley RS, D. E., "Gold nanoparticle-mediated photothermal therapy: applications and opportunities for multimodal cancer treatment," Wiley Interdiscip Rev Nanomed Nanobiotechnol. 9(4) (2017).

[3] A.A. Popov, e. a., "Laser- synthesized tin nanoparticles as promising plasmonic alternative for biomedical applications," Scientific Reports (In press).

[4] H. Ke, S. Tai, L. W., "Photoacoustic thermography of tissue," Journal of Biomedical Optics 19 (2014). 